\title{
Role of Gamma Knife surgery in the management of pineal region tumors
}

\author{
Gregory P. Lekovic, M.D., Ph.D., J.D., ${ }^{1}$ L. Fernando GonZalez, M.D., ${ }^{1}$ \\ Andrew G. Shetter, M.D., ${ }^{1}$ Randall W. Porter, M.D., ${ }^{1}$ KRis A. SMith, M.D., ${ }^{1}$ \\ DaVII Brachman, M.D., ${ }^{2}$ AND Robert F. SPETZler, M.D. ${ }^{1}$ \\ ${ }^{1}$ Division of Neurological Surgery, Barrow Neurological Institute; and ${ }^{2}$ Department of Radiation \\ Oncology, St. Joseph's Hospital and Medical Center, Phoenix, Arizona
}

\begin{abstract}
Object. Increasingly, radiosurgery is used to treat pineal region tumors, either as a primary treatment or as an adjunct to conventional radiation therapy. The authors report their experience with Gamma Knife surgery (GKS) for the treatment of pineal region tumors.

Methods. The authors retrospectively reviewed the charts of all patients undergoing GKS at their institution between 1997 and 2005. Seventeen patients underwent GKS for nonmetastatic tumors of the pineal region. All patients were treated using Leksell Gamma Plan treatment planning software (versions 4.12::5.34). The mean treatment volume was $7.42 \mathrm{~cm}^{3}$ (range 1.2-32.5 $\mathrm{cm}^{3}$ ). Prescribed doses ranged from 12 to $18 \mathrm{~Gy}$. All doses were prescribed to the $50 \%$ isodose line. Independent neuroradiologists reviewed all follow-up imaging studies for evidence of progression of disease.

Results. One patient (Case 10) died 6 days after GKS. Mean clinical and imaging follow-up in the remaining 16 cases was 31 months. Local control was established during a mean neuroimaging follow-up period of 31 months (range 1-95) in 16 patients (100\%). In 2 of these 16 patients (one with an anaplastic astrocytoma, the other with a primitive neuroectodermal tumor), leptomeningeal and spinal spread of tumor developed despite control of the pineal lesions. There were no new neurological deficits attributable to GKS. Three patients died (including the one who died 6 days after GKS) during the follow-up period.

Conclusions. Excellent control of pineal region brain tumors can be obtained with GKS when it is used in conjunction with surgery, conventional radiation therapy, or both. Patient survival and quality of life can be optimized through the use of multimodal treatment, including surgery, conventional radiation therapy and/or radiosurgery, and chemotherapy, when applicable. (DOI: 10.3171/FOC-07/12/E12)
\end{abstract}

\section{Key WORDS • Gamma Knife surgery • germ cell tumor • pineal region tumor • pineoblastoma • pineocytoma $\bullet$ radiosurgery}

$\mathrm{T}$ HE role of radiosurgery in the treatment of tumors of the pineal region is controversial. The argument for radiosurgery is that the highly conformal treatment planning possible with radiosurgery allows delivery of cytotoxic doses of radiation to the target field while minimizing the dose delivered to neighboring vital structures. Hence, radiosurgery may be a less morbid alternative to treatment for these deep-seated lesions surrounded by eloquent structures. The argument against radiosurgery is

Abbreviations used in this paper: EBRT = external-beam radiation therapy; ETV = endoscopic third ventriculostomy; GKS = Gamma Knife surgery; GTR = gross-total resection; IMRT = intensity-modulated radiation therapy; $\mathrm{MR}=$ magnetic resonance; NGGCT $=$ nongerminomatous germ cell tumor; PNET $=$ primitive neuroectodermal tumor; $\mathrm{VP}=$ ventriculoperitoneal. twofold. First, for tumors with known radiosensitivity, radiosurgery is unnecessary. Second, for relatively radioresistant lesions (that is, low-grade lesions such as pineocytomas), GTR is curative and can usually be achieved with minimal complications.

Nevertheless, radiosurgery is being used increasingly to treat pineal region tumors, either as the principal treatment modality ${ }^{8,9}$ or in conjunction with conventional EBRT. ${ }^{2} \mathrm{We}$ report our series of 17 nonmetastatic tumors of the pineal region treated with GKS.

\section{Clinical Material and Methods}

The charts of all patients undergoing GKS of the pineal region at our institution between 1997 and 2005 (25 patients) were reviewed retrospectively. Three cases of arteriovenous malformations and 5 involving metastases from known extracranial disease were excluded from analysis. 
TABLE 1

Clinical summary of 17 cases of pineal region tumors*

\begin{tabular}{|c|c|c|c|c|c|c|c|c|c|}
\hline $\begin{array}{l}\text { Case } \\
\text { No. }\end{array}$ & $\begin{array}{l}\text { Age, } \\
\text { Sex }\end{array}$ & Diagnosis & \multicolumn{4}{|c|}{ Dosimetry } & $\begin{array}{l}\text { Duration of } \\
\text { Clinical FU }\end{array}$ & Response & $\begin{array}{l}\text { Complications } \\
\text { or Death }\end{array}$ \\
\hline 2 & $61, \mathrm{M}$ & pineocytoma & 12.4 & 13 & 17 & 26 & $2 \mathrm{mos}$ & $\begin{array}{l}\mathrm{PR} \text { at } 2 \operatorname{mos}(75 \% \\
\text { decrease in size })\end{array}$ & $\begin{array}{l}\text { died } 2 \text { mos } \\
\text { after GKS }\end{array}$ \\
\hline 3 & $62, \mathrm{M}$ & pineocytoma & 2.6 & 14 & 12 & 28 & $16 \mathrm{mos}$ & no cx at $15 \mathrm{mos}$ & none \\
\hline 7 & $37, \mathrm{~F}$ & pineocytoma & 5.2 & 14 & 7 & 28 & $6 \mathrm{mos}$ & $\mathrm{PR}$ at $6 \mathrm{mos}$ & none \\
\hline 8 & $24, \mathrm{M}$ & pineocytoma & 1.99 & 15 & 8 & 30 & $6 \mathrm{mos}$ & no cx at $13 \mathrm{mos}$ & none \\
\hline 9 & $55, \mathrm{~F}$ & $\begin{array}{l}\text { choroid plexus } \\
\text { adenoma }\end{array}$ & 3.9 & 16 & 11 & 32 & $96 \mathrm{mos}$ & $\mathrm{PR}$ at $95 \mathrm{mos}$ & none \\
\hline 10 & $78, \mathrm{~F}$ & $\begin{array}{l}\text { pineal paren- } \\
\text { chymal tumor } \\
\text { of intermed diff }\end{array}$ & 32.5 & 12 & 27 & 24 & 6 days & none & $\begin{array}{l}\text { died } 6 \text { days } \\
\text { after GKS }\end{array}$ \\
\hline 14 & $55, \mathrm{M}$ & PNET & 8.3 & 12 & 10 & 24 & $44 \mathrm{mos}$ & $\begin{array}{l}\text { distant progr at } 35 \text { mos, } \\
\text { PR at } 44 \text { mos } \\
\text { ( } 5 \text { mos after } \\
\text { retreatment) }\end{array}$ & $\begin{array}{l}\text { distant } \\
\text { treatment } \\
\text { failure }\end{array}$ \\
\hline 15 & $13, \mathrm{M}$ & NGGCT & 1.2 & 15 & 8 & 30 & $73 \mathrm{mos}$ & $\mathrm{CR}$ at $73 \mathrm{mos}$ & none \\
\hline 16 & $27, \mathrm{~F}$ & $\begin{array}{l}\text { low-grade } \\
\text { astrocytoma }\end{array}$ & 1.6 & 14 & 8 & 28 & $21 \mathrm{mos}$ & $\mathrm{CR}$ at $21 \mathrm{mos}$ & none \\
\hline 17 & $22, \mathrm{M}$ & pineoblastoma & 23.0 & 14 & 15 & 28 & $45 \mathrm{mos}$ & no cx at $45 \mathrm{mos}$ & none \\
\hline
\end{tabular}

$* \mathrm{CR}=$ complete response; $\mathrm{cx}=$ change; diff = differentiation; $\mathrm{FU}=$ follow-up; intermed = intermediate; $\mathrm{PR}=$ partial response; progr = progression.

Seventeen patients (10 male and 7 female) underwent GKS for pineal region tumors other than metastatic disease (Table 1). Their mean age was 43.8 years (range 13-78 years).

Histopathological diagnosis was obtained in 15 cases by open biopsy via a supracerebellar infratentorial approach. Twelve patients underwent open biopsy as their initial procedure. Endoscopic biopsy was followed by attempted resection and open biopsy in 3 cases (1 PNET, 1 NGGCT, and 1 low-grade astrocytoma). However, only one of these attempts was related to a nondiagnostic endoscopic biopsy. In two cases diagnosis was made by endoscopic biopsy only. No diagnoses were made by stereotactic biopsy.

The patient with a PNET lesion (Case 14) presented for GKS 9 years after his initial tumor resection; upon recurrence, only an endoscopic biopsy was performed before he underwent GKS.

Clinical follow-up data were obtained by chart review of a patient's most recent follow-up visit or by telephone. One patient (Case 10) died 6 days after GKS, and this case was excluded from the follow-up analysis. Follow-up imaging studies for the 16 remaining patients were evaluated by independent neuroradiologists for evidence of disease progression. Local control was defined as no change, partial response, or complete response.

\section{Radiosurgery Technique}

All patients were treated using Leksell Gamma Plan treatment planning software (versions 4.12-5.34, Elekta). The mean prescription dose was 14.06 Gy (range 12-18 Gy). The median and mode dose was also 14 Gy. All doses were prescribed to the $50 \%$ isodose line. The mean target volume was $7.42 \mathrm{~cm}^{3}\left(\right.$ range $\left.1.2-32.5 \mathrm{~cm}^{3}\right)$. Between 7 and 27 isocenters (mean 11.4 isocenters) were used in treatment planning. Whenever possible, the Automated Positioning System (Elekta) was used to obtain highly conformal treatment plans.

Fourteen patients were treated with GKS as either the primary radiation modality or as a salvage therapy for a recurrence after conventional EBRT failed. Two patients, one with an NGGCT and another with an anaplastic astrocytoma, were administered a 15-Gy and 12-Gy GKS boost to the pineal region after receiving reduced-dose craniospinal radiation therapy (3600 cGy).

\section{Results}

Previous therapies (that is, interventions other than biopsy) included attempted tumor resection and/or debulking in 16 cases, IMRT in 3 cases ( 2 at a reduced dose with planned GKS boost), and chemotherapy with reduced-dose (3600 cGy) craniospinal radiation therapy in 3 cases (Table 2). Two patients (Cases 3 and 5) had undergone repeated craniotomy for recurrent tumor (4 years and 1 year after initial surgery, respectively). One patient had undergone a single craniotomy at another institution (Case 2). Another patient 
TABLE 2

Previous therapies in 17 patients with pineal tumors

\begin{tabular}{lc}
\hline \multicolumn{1}{c}{ Treatment } & Value* \\
\hline craniotomy & \\
supracerebellar-infratentorial & 17 \\
interhemispheric & 2 \\
endoscopic approach & 5 \\
biopsy & 5 \\
ETV & 1 \\
resection & 13 \\
VP shunt & \\
chemotherapy & 1 \\
carboplatin/mesna/cytotaxin/bleomycin & 1 \\
temozolomide & 1 \\
etoposide/cyclophosphamide/mesna & \\
radiation & 3 \\
3600 cGy craniospinal & $3 \dagger$ \\
IMRT & \\
\hline
\end{tabular}

* Values represent numbers of therapeutic interventions, with a course of chemotherapy considered as a single intervention. The sum exceeds 17 because most patients underwent multiple therapeutic interventions.

$\dagger$ Two with planned GKS boost.

had undergone multiple resections, both open and endoscopic, at another institution (Case 13). One patient (Case 11) had had no previous treatment other than endoscopic biopsy. Another patient (Case 7) underwent a GTR via a supracerebellar infratentorial approach and was treated with GKS to the tumor bed for presumed microscopic disease. Thus, 14 patients presented for GKS as a salvage treatment for residual or recurrent disease after attempted resection, biopsy, or previous whole-brain irradiation.

Sixteen patients underwent cerebrospinal fluid diversion procedures before GKS (5 ETVs and 11 VP shunt placements). Despite attempts to manage hydrocephalus with ETV, all patients but one (Case 11) eventually required a VP shunt. Two patients who initially underwent ETV required VP shunt placement before GKS while 2 others required VP shunt placement after GKS.

The time to GKS from initial diagnosis, treatment, or both ranged from 2 weeks to 16 years. Although 16 patients had undergone a previous resection, in 2 cases GKS was performed only after attempted repeated resection (Cases 3 and 5).

In another case (Case 10), the patient's condition was diagnosed at an outside institution, and the patient was treated with VP shunts and followed up for 16 years before MR imaging revealed evidence of progression. Upon resection ( 8 weeks before GKS) at our institution, the tumor was found to be a pineocytoma of mixed differentiation, with a moderately elevated MIB- 1 index. The original treatment plan for this patient included postoperative IMRT. The patient's clinical condition was declining rapidly, however, and the decision was made to proceed with GKS in lieu of IMRT. Despite the GKS, the patient died 6 days after treatment. This patient was therefore excluded from follow-up analysis.

A complete imaging response was obtained in 2 patients and a partial response in an additional 8. Local control (defined as no change or partial or complete response) was established with a mean imaging follow-up of 31 months (range 1-95 months) in 16 patients (100\%). In 1 patient with a malignant mixed germ cell tumor (Case 15), a new $1-\mathrm{cm}$ nodule of enhancement was found to have developed in the treatment field 9 months after treatment. Previous interval imaging studies had demonstrated complete response of the tumor. The patient has been followed up with serial imaging. On the 73-month follow-up images, there was no longer evidence of enhancement. As of this writing, the patient has not received treatment for 5 years. In this case, the transient imaging finding may have represented a treatment effect.

In 2 patients (one with an anaplastic astrocytoma, the other with a PNET), leptomeningeal and spinal spread of tumor developed despite control of the pineal lesion. The patient with an anaplastic astrocytoma was treated with 5940 cGy IMRT with a GKS boost of 12 Gy to the pineal region, but leptomeningeal and spinal metastases subsequently developed. Palliative craniospinal irradiation and chemotherapy were initiated, but 8 months after GKS the treatment was discontinued because of the patient's poor tolerance of the side effects, and the patient died. The patient with a PNET underwent repeated GKS for 7 ventricular and suprasellar metastases and CyberKnife stereotactic radiosurgery for a chiasmatic lesion 35 months after initial GKS treatment. At the most recent follow-up (10 months), all lesions had responded to the repeated radiosurgery (Fig. 1).

The mean duration of clinical follow-up was 31 months (range 1-96 months). There were no new neurological deficits attributable to GKS. There were 3 postoperative complications. One intracerebral hemorrhage required evacuation after an infratentorial-supracerebellar approach. There was one perioperative stroke and one intraventricular hemorrhage followed endoscopic biopsy. No complications were attributable to GKS.

Three patients died. One (Case 10) died 6 days after GKS and thus was excluded from further analysis. The second patient (Case 2) died 2 months after GKS; however, MR images obtained immediately before the patient's death showed a $75 \%$ reduction in the size of the lesion. The third patient (Case 12) experienced widespread distant metastases from his anaplastic astrocytoma; this patient's family elected to withdraw further care, and the patient died 8 months after GKS.

\section{Discussion}

The role of radiosurgery in the management of pineal region tumors remains controversial. Traditionally, pineal region tumors have either been approached surgically, with the goal of GTR and cure (if benign), or with conventional radiation, if the tumor type is known to be radiosensitive, malignant, or both. In contrast, radiosurgery has the potential to be used either as a "boost" modality in conjunction with conventional radiotherapy or as an alternative to radiotherapy, surgery, or both.

We have recently reviewed the English-language literature on the use of radiosurgery for the treatment of pineal region tumors. Nine such published reports were identified, including 97 cases. ${ }^{1-9}$ Several factors, however, make it difficult to compare these studies to our study: small sample size, heterogeneity of diagnoses, and differences in radiosurgical techniques. 
G. P. Lekovic et al.
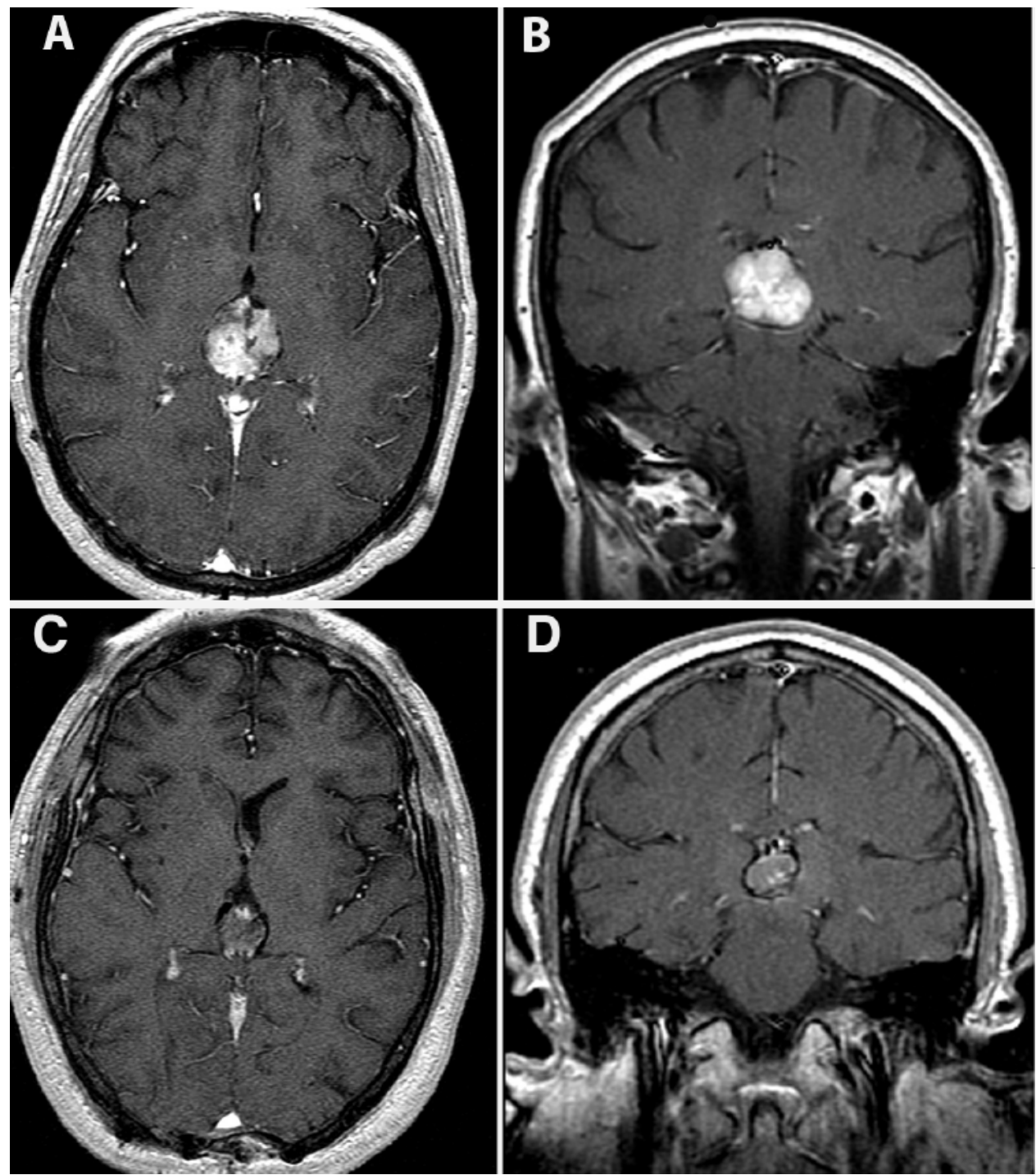

FIG. 1. Magnetic resonance images obtained before (A and B) and after (C and D) GKS retreatment for the distant recurrence of a PNET 35 months after the initial lesion was treated. Note the absence of enhancement in the pineal region.

Perhaps the most significant issue in comparing our data to that of others is the heterogeneity of tissue diagnosis and tumor grade in our population of patients. Because of the histological heterogeneity of pineal region tumors and their relative lack of diagnostic specificity on preoperative imaging modalities, biopsy (whether stereotactic, endoscopic, or open) must be performed to obtain a tissue diagnosis before definitive treatment is instituted. We do not believe that empirical treatment of pineal region tumors with radiosurgery is justified, as the treatment paradigm is critically dependent upon tumor histological grade. For high-grade lesions, GKS may be more appropriate as a boost modality than as a primary treatment, while for low-grade lesions GKS may suffice as a stand-alone therapy. All 17 of our patients had undergone biopsy, and most had undergone a craniotomy for tumor debulking or resection. Four patients initially had undergone endoscopic biopsy followed by craniotomy.

The ideal means of obtaining a tissue diagnosis in pa- tients harboring pineal region tumors is controversial. Open surgery, as opposed to stereotactic surgery or endoscopic biopsy, has been advocated as a superior means of obtaining accurate tissue diagnoses because of enhanced visualization of the tumor. A more representative sampling of the tumor can therefore be obtained for histopathological diagnosis. In our experience, in only one case was the need for open biopsy attributed to nondiagnostic sampling during attempted endoscopic biopsy.

Although minimally invasive approaches to the pineal region (for example, endoscopy followed by GKS) are not the focus of this study, we have found them to be disappointing. We initially chose an endoscopic approach to the treatment of 5 patients with pineal region masses; in only 2 cases (Cases 8 and 11) did the patient avoid open craniotomy and/or EBRT. The lesions in these cases were diagnosed endoscopically, and we proceeded directly to treatment with GKS. The ETV failed in one of these 2 cases, however, and VP shunt placement was required. In fact, VP 
shunt placement was required in all but one of the cases initially managed with an ETV. The exception was Case 11.

We have favored a more invasive approach (open biopsy with attempted GTR or debulking). Nevertheless, this series, by definition, only includes cases in which patients underwent GKS, and therefore cases managed successfully by minimally invasive approaches with adjuvant EBRT or cases in which patients underwent GTR without any adjuvant therapy have been excluded.

Many authors have advocated a management algorithm for pineal region lesions that includes attempted GTR. Even in cases of malignant or radiosensitive tumors, debulking may be beneficial. ${ }^{10}$ We observed no neurological benefit attributable to tumor debulking, although this was not an endpoint of the present study. Furthermore, although we have demonstrated high rates of tumor control with radiosurgery, we believe that craniotomy and GTR are the treatment modalities of choice for benign lesions or those thought to be benign. Only craniotomy and GTR offer the possibility of a definitive cure, given that the majority of patients in our series underwent craniotomy for open biopsy and hence incurred the attendant risks, we believe that a judicious attempt at GTR is warranted. As mentioned, in our opinion the role of endoscopy remains unclear. If GTR is impossible or can only be achieved with an unsatisfactory risk to the patient, then radiosurgery for residual or recurrent tumors offers a highly efficacious and minimally morbid alternative. Nevertheless, our data do support the use of GKS as a primary treatment modality for those patients in whom an adequate tissue diagnosis of a lowgrade neoplasm has been made via endoscopy or stereotactic biopsy.

Conventional radiation therapy is highly effective in the treatment of germ cell tumors and therefore of most pineal region tumors. Nonetheless, concerns about radiation toxicity, especially with regard to intellect and to the function of the hypothalamic-pituitary axis in children, have prompted efforts to reduce or avoid conventional radiation therapy. For the treatment of germ cell tumors, these efforts have led to the adoption of chemotherapy regimens shown to be efficacious in the treatment of gonadal germ cell tumors. Intracranial germ cell tumors, however, are not amenable to treatment with chemotherapy alone; they can be treated successfully with a combination of chemotherapy and reduced-dose radiation. The rationale for radiosurgery for radiation-sensitive tumors, either as a boost to reduce the dose of conventional radiation or to replace it, is similar to that of chemotherapy (that is, to avoid, delay, or reduce the dose of conventional EBRT).

We used radiosurgery both as an independent adjuvant therapy and in conjunction with conventional EBRT/IMRT in the management of malignant pineal region tumors, including malignant mixed germ cell tumor, anaplastic astrocytoma, PNET, and pineoblastoma. Unfortunately, both of our patients with malignant lesions who were treated with GKS in lieu of EBRT (as opposed to being treated with GKS as a boost modality) developed ventricular-leptomeningeal and spinal metastases after radiosurgery even though local tumor control was achieved. Despite the small sample size, we conclude that radiosurgery may be a useful alternative to conventional EBRT/IMRT for the treatment of these lesions, given the possibility of salvage treatment with either repeated GKS or EBRT. Radiosurgery must, however, be used with caution if it is to be used as a primary treatment for malignant disease.

\section{Conclusions}

We obtained excellent control of pineal region brain tumors with GKS when we used it in conjunction with surgery, conventional radiation therapy, or both. Because of the importance of histopathological characteristics in the natural history of these tumors, the specific treatment algorithm must be tailored to the individual patient. Overall, we believe that benign tumors are best approached surgically, with GKS being ideally suited to the treatment of residual or recurrent disease. We recognize that our mean follow-up (31 months) may still be too brief to demonstrate conclusively the efficacy of GKS in the management of slowgrowing, benign tumors. We also used GKS successfully in conjunction with reduced-dose craniospinal radiation therapy in the treatment of malignant pineal region tumors when GKS was planned as a boost modality. Based on our experience, we believe that multimodal treatment, including surgery, conventional radiation and/or radiosurgery, and chemotherapy (when applicable), provides the most effective strategy for optimizing patient survival and quality of life.

\section{References}

1. Backlund EO, Rahn T, Sarby B: Treatment of pinealomas by stereotaxic radiation surgery. Acta Radiol Ther Phys Biol 13: 368-376, 1974

2. Casentini L, Colombo F, Pozza F, Benedetti A: Combined radiosurgery and external radiotherapy of intracranial germinomas. Surg Neurol 34:79-86, 1990

3. Dempsey PK, Lunsford LD: Stereotactic radiosurgery for pineal region tumors. Neurosurg Clin N Am 3:245-253, 1992

4. Deshmukh VR, Smith KA, Rekate HL, Coons S, Spetzler RF: Diagnosis and management of pineocytomas. Neurosurgery 55: 349-357, 2004

5. Hasegawa T, Kondziolka D, Hadjipanayis CG, Flickinger JC, Lunsford LD: The role of radiosurgery for the treatment of pineal parenchymal tumors. Neurosurgery 51:880-889, 2002

6. Hasegawa T, Kondziolka D, Hadjipanayis CG, Flickinger JC, Lunsford LD: Stereotactic radiosurgery for CNS nongerminomatous germ cell tumors. Report of four cases. Pediatr Neurosurg 38:329-333, 2003

7. Kobayashi T, Kida Y, Mori Y: Stereotactic gamma radiosurgery for pineal and related tumors. J Neurooncol 54:301-309, 2001

8. Manera L, Régis J, Chinot O, Porcheron D, Levrier O, Farnarier P, et al: Pineal region tumors: the role of stereotactic radiosurgery. Stereotact Funct Neurosurg 66 (1 Suppl):164-173, 1996

9. Subach BR, Lunsford LD, Kondziolka D: Stereotactic radiosurgery in the treatment of pineal region tumors. Prog Neurol Surg 14:175-194, 1998

10. Weiner HL, Finlay JL: Surgery in the management of primary intracranial germ cell tumors. Childs Nerv Syst 15:770-773, 1999

Manuscript submitted September 24, 2007.

Accepted October 15, 2007.

Address correspondence to: Andrew G. Shetter, M.D., c/o Neuroscience Publications, Barrow Neurological Institute, 350 West Thomas Road, Phoenix, Arizona 85013. email: neuropub@ chw.edu. 